\title{
Soy intake and breast cancer risk: a prospective study of 300,000 Chinese women and a dose-response meta-analysis
}

\author{
Yuxia Wei ${ }^{1} \cdot$ Jun $\mathrm{Lv}^{1,8,9} \cdot \mathrm{Yu} \mathrm{Guo}^{2} \cdot$ Zheng Bian ${ }^{2} \cdot$ Meng Gao ${ }^{1} \cdot$ Huaidong $\mathrm{Du}^{3,4} \cdot$ Ling Yang $^{3,4} \cdot$ Yiping Chen $^{3,4}$. \\ $\mathrm{Xi} \mathrm{Zhang}^{5}$. Tao Wang ${ }^{5}$. Junshi Chen ${ }^{6}$. Zhengming Chen ${ }^{4}$. Canqing Yu ${ }^{1}$. Dezheng Huo ${ }^{7}{ }^{\circ} \cdot$ Liming Li $^{1}$ on behalf of \\ the China Kadoorie Biobank Collaborative Group
}

Received: 9 July 2019 / Accepted: 13 November 2019 / Published online: 21 November 2019

(c) The Author(s) 2019

\begin{abstract}
Epidemiological evidence on the association of soy intake with breast cancer risk is still inconsistent due to different soy intake levels across previous studies and small number of breast cancer cases. We aimed to investigate this issue by analyzing data from the China Kadoorie Biobank (CKB) study and conducting a dose-response meta-analysis to integrate existing evidence. The CKB study included over 300,000 women aged 30-79 from 10 regions across China enrolled between 2004 and 2008, and followed-up for breast cancer events until 31 December 2016. Information on soy intake was collected from baseline, two resurveys and twelve 24-h dietary recalls. We also searched for relevant prospective cohort studies to do a dose-response meta-analysis. The mean (SD) soy intake was $9.4(5.4) \mathrm{mg} /$ day soy isoflavones among CKB women. During 10 years of follow-up, 2289 women developed breast cancers. The multivariable-adjusted relative risk was $1.00(95 \%$ confidence interval [CI] $0.81-1.22)$ for the fourth (19.1 mg/day) versus the first (4.5 mg/day) soy isoflavone intake quartile. Meta-analysis of prospective studies found that each $10 \mathrm{mg} /$ day increment in soy isoflavone intake was associated with a $3 \%$ (95\% CI 1-5\%) reduced risk of breast cancer. The CKB study demonstrated that moderate soy intake was not associated with breast cancer risk among Chinese women. Higher amount of soy intake might provide reasonable benefits for the prevention of breast cancer.
\end{abstract}

Keywords Soy intake $\cdot$ Breast cancer $\cdot$ Prospective cohort study $\cdot$ Dose-response meta-analysis

\section{Introduction}

Breast cancer is the most common cancer and one of the leading causes of cancer death among women throughout the world [1]. Its incidence rate varies across regions, which is much higher in Western than in Asian countries [1-5]. This regional variation has been postulated to be attributed to

The members of steering committee and collaborative group are listed in the Acknowledgements.

Electronic supplementary material The online version of this article (https://doi.org/10.1007/s10654-019-00585-4) contains supplementary material, which is available to authorized users.

Canqing Yu

yucanqing@pku.edu.cn

$\bowtie$ Dezheng Huo

dhuo@health.bsd.uchicago.edu

Extended author information available on the last page of the article the differences in lifestyle and dietary factors. One of such dietary components is soy foods, which are staples in Asian diet but rare in diet of western populations. Soy foods are major sources of dietary isoflavones, which share structural similarities with $17-\beta$-estradiol and may serve as functional estrogen antagonists to protect against breast cancer [6,7]. Also, soy isoflavones may exert effect through estrogen independent pathways [6].

Epidemiological evidence on the relationship between soy foods and breast cancer is still limited and inconclusive [8], despite potential mechanisms from experiments [6, 7]. Several meta-analyses concluded that higher amount of soy intake was associated with lower risk of incident breast cancer among Asian women but not among their Western counterparts [9-12]. However, between-study heterogeneity was obvious [9-12] and most original studies included in these meta-analyses were case-control studies [10-12]. By contrast, a recent meta-analysis involving only cohort studies, which are less subject to selection and recall bias, found 
no association between isoflavone intake and breast cancer, but it showed that a higher consumption of soy-based foods was weakly associated with a lower risk of breast cancer relative to a low consumption of soy foods (HR: 0.87, 95\% CI 0.76-1.00) [13]. The definition of "high" soy food intake varies across studies in previous meta-analyses, making it difficult to interpret the pooled risk estimates. Meta-analysis taking into account the amount of soy foods or isoflavone dose is desirable.

Several cohort studies or nested case-control studies related to soy and incident breast cancer gave inconsistent results. Studies conducted in Western countries where dietary soy intake levels were low [14-24] or moderate [25, 26] have found no clear association. Evidence from studies in Asia where dietary soy intake levels were moderate to high was also inconsistent. Some studies found no statistically significant association $[27,28]$ while others reported a reduced breast cancer risk for women in the highest soy intake groups [29-32]. As most previous cohort studies in Asia have limited numbers of breast cancer cases, insufficient statistical power as well as different cut-off values for soy intake categories may explain this inconsistency. To our knowledge, only one cohort study, namely the Shanghai Women's Health Study (SWHS) which enrolled participants from a highly developed city, was conducted in China previously [32]. More evidence from the general Chinese adults with diverse economic background is still lacking.

Using the data from the China Kadoorie Biobank (CKB), a large scale prospective cohort study involving over 300,000 women from 10 geographically and economically diverse regions in China, we evaluated the relationship between soy intake and risk of incident breast cancer. Subgroup analyses were also conducted to assess whether risk estimates varied by baseline characteristics like menopausal status. Participants' soy consumption levels and cut-off values of soy categories varied across previous studies. In order to provide a clearer picture on the soy-breast cancer relationship, we also did a dose-response meta-analysis to integrate results of prospective cohort studies.

\section{Methods}

\section{Study population}

The CKB study recruited participants from 5 urban areas and 5 rural areas in China. The study design and methods have been described in detail elsewhere [33]. Briefly, between June 2004 and July 2008, the study enrolled 512,715 adults (302,510 women) aged 30-79 years who completed baseline data collection, including a questionnaire and physical measurements after signing a written informed consent form. Among the women recruited at baseline, we excluded persons with previously cancer diagnoses $(n=1610)$, with missing values for key variables $(n=47$ for reproductive characteristics, $\mathrm{n}=1$ for body mass index [BMI]), leaving 300,852 female participants in the main analyses. Approval of the study was obtained from ethics committees or institutional review boards at the University of Oxford, the Chinese Center for Disease Control and Prevention, the Chinese Academy of Medical Sciences, and all participating centers.

\section{Assessment of soy consumption}

In the baseline questionnaire, one item asked participants about the overall frequency of soy foods (e.g. soybeans, fresh tofu, fried tofu, pressed tofu, soymilk skin or film, soybean flakes, soymilk and so on) during the past 12 months: never/ rarely, monthly, 1-3 days per week, 4-6 days per week, or daily. After participants completed the baseline survey, two resurveys were conducted in 2008 and 2013, respectively, each involving about $5 \%$ of the randomly selected participants from each of the 10 study regions. The first resurvey questionnaire asked exactly the same question on soy consumption as the baseline one, whereas the question was split into 2 items in the second resurvey. One item asked about the frequency and amount of soymilk consumption and the other item about soy foods other than soymilk. To evaluate the reproducibility and validity of food frequency questionnaires (FFQs) used in baseline and resurveys, an intensive dietary study of 432 CKB participants (254 women) was conducted from 2015 to 2016. These participants completed two FFQs (median interval: 3.3 months) and twelve 24-h dietary recalls (24-HDR). The 24-HDRs covered all kinds of soy foods in China and were conducted in three seasons separately, with 3 weekdays and 1 weekend day in each season. For female participants, the weighted Kappa statistic was 0.66 for reproducibility of baseline soy food frequency and 0.77 for reproducibility of soy consumption amount (excluding soy milk) in $2^{\text {nd }}$ resurvey. Using 24-HDRs as the gold standard, the weighted Kappa statistic was 0.67 for validity of baseline food frequency and 0.74 for validity of soy consumption amount (excluding soy milk) in $2^{\text {nd }}$ resurvey.

We combined intake frequency from baseline and 1st resurvey and the intake amount from the 2 nd resurvey to estimate the usual amount of soy intake for each woman. Then, we converted the usual amount of soy intake into amount of soy isoflavone by taking into account the proportions that different kinds of soy foods contributing to total soy food amount among women in the 24-HDRs and the soy isoflavone content of different soy foods (Appendix).

\section{Assessment of covariates}

Information on socio-demographic characteristics (age, education and household income), lifestyle factors (alcohol 
consumption, physical activity and dietary habits), reproductive characteristics (i.e. age at menarche, parity, breastfeeding duration, menopausal status, menopausal age, and use of oral contraceptives), and family history of cancer were obtained from the baseline questionnaire. Daily energy intake was calculated by taking into account the 12 groups of foods available in the present study. A participant was considered as having a family history of cancer if at least one of their parents or siblings was diagnosed with cancer. At baseline, body weight and height were measured by trained staff using calibrated instruments and BMI was calculated by weight $(\mathrm{kg}) /$ height squared $\left(\mathrm{m}^{2}\right)$.

\section{Identification of breast cancer cases}

Participants were followed-up from the date of completing baseline questionnaire to the date of diagnosis of breast cancer, death, loss to follow-up or 31 December 2016, whichever came first. By 31 December 2016, about 1\% participants were censored due to loss to follow-up. Incident breast cancer cases were identified periodically through linkage with local disease and death registries [34], and the national health insurance system or ascertained through active follow up. All diseases were coded according to the International Classification of Diseases, 10th Revision (ICD-10), by trained staff blinded to baseline information of participants. Breast cancer was coded as $\mathrm{C} 50$. The proportion of death certificate only cases was $4.1 \%$ for breast cancer, indicating the high completeness of cancer registration in the present study.

\section{Statistical analysis}

Baseline characteristics of participants were presented as means (standard deviations, SDs) or percentages across 4 categories of baseline soy consumption, standardized for age and study region if appropriate, via logistic regressions for categorical variables or multiple linear regressions for continuous variables.

Hazard ratios (HRs) and 95\% confidence intervals (95\% CIs) were calculated for breast cancer risk by both soy frequency and usual amount quartiles using Cox proportional hazard regression models. Categorical soy intake variables were treated as continuous variables to evaluate the linear trend. In the Cox models, age was used as the underlying time scale and baseline characteristics as covariates. Each Cox model was stratified by baseline age groups (in 5-year intervals) and study region. Potential confounding factors adjusted for in the multivariable models were: education attainment, household income, smoking status, alcohol consumption, physical activity, baseline BMI, standing height, age at menarche, parity, average breastfeeding duration, menopausal status and age at menopause, use of oral contraceptives, family history of cancer, total energy intake, and consumption frequency of fresh fruit, fresh vegetables, preserved vegetables, red meat, poultry, fish and dairy products at baseline. Information on hormone replacement therapy was not collected at baseline and thus was not adjusted for in the models. However, we believed it would not influence the risk estimates since $<1 \%$ of the females have ever used hormone replacement treatment according to data from the second resurvey. The method of Schoenfeld residuals found no violation of the proportional hazards assumption for soy intake in fully-adjusted model.

A stratified analysis was further performed separately among pre-menopausal and post-menopausal women to assess any modifying effect of menopause on the association of soy consumption and breast cancer. Besides, stratified analyses were conducted according to other baseline characteristics such as BMI. Multiplicative interactions were tested using likelihood ratio tests comparing models with and without the cross product terms between stratifying variables and soy food consumption categories.

Several sensitivity analyses were done separately: (1) by excluding females who developed breast cancer during the first 2 years of follow-up; or (2) by excluding those with a family history of cancer.

\section{Systematic review and dose-response meta-analysis}

We searched on PubMed, Embase and Cochrane library from their dates of inception to March 2019 for prospective studies examining the association between soy intake and breast cancer. Studies using concentrations of isoflavones or their metabolites in biological samples were not included owing to the difficulty in converting biological concentration into amount of soy isoflavone intake. However, we believed that this had no great impact on the result because studies using biological concentrations as exposure assessment were mainly conducted among populations with pretty low level of soy intake $[14,16,19]$. We excluded studies if the amount of soy intake was unavailable and could not be estimated using relevant data. We also excluded reviews, non-human studies, abstract-only publications or editorials. If the same cohort study published more than one original articles on soy intake and breast cancer, the paper reporting the largest sample size, longest follow-up time, or the widest variation in soy intake levels was kept. In addition, studies in which participants' soy intake levels were very low (i.e. mean or median intake $<5 \mathrm{mg} /$ day of soy isoflavone for the highest consumption group) were not included in the quantitative synthesis (dose-response meta-analysis) as the weights contributed by these studies were negligible. Detailed literature searching strategy, data extraction methods, and quality assessment of individual study were presented in Appendix. 
In the dose-response meta-analysis, HRs were considered as effect sizes and the median or mean soy isoflavone intake of each consumption category was regarded as the consumption dose of corresponding category. To test for potential non-linear relationship between soy isoflavone intake and incident breast cancer, a non-linear dose-response meta-analysis was done by coding soy isoflavone intake as a restricted cubic spline (RCS) function. If there was no evidence of non-linear association, we firstly estimated study-specific log HRs and 95\% CIs for each $10 \mathrm{mg} /$ day increment in soy isoflavone intake using the method introduced by Greenland and Longnecker [35]. Then we pooled study-specific log HRs to obtain a summarized effect size using a fixed effect model. Between-study heterogeneity was assessed by $\mathrm{I}^{2}$ statistic and the Egger test was used to detect publication bias.

Several sensitivity analyses were done to assess the robustness of dose-response meta-analysis: (1) dropping one of the studies included in the main dose-response meta-analysis each time; (2) dropping studies grouping participants according to soy frequency rather than amount of soy intake; (3) including only those studies which assessed soy intake in a more precise way (i.e. assessing both frequency and amount of soy intake using validated FFQs at baseline); or (4) including all the studies in the synthetic review, i.e. additionally including studies with extremely low level of soy isoflavone intake.

All statistical analyses were performed with Stata (version 15). All $P$ values were two-sided and statistical significance was defined as $P<0.05$.

\section{Result}

\section{Patterns of soy consumption}

Of the 300,852 female participants, $44.5 \%$ resided in urban areas, and the mean (SD) age at baseline was 50.9 (10.5) years (Table 1). At baseline, $12.0 \%$ reported never/rarely consuming soy foods (non-consumers) and $9.3 \%$ reported

Table 1 Baseline characteristics of participants by baseline frequency of soy consumption ${ }^{\mathrm{a}}$

\begin{tabular}{|c|c|c|c|c|c|}
\hline & $\begin{array}{l}\text { Never } \\
\text { or rarely } \\
(n=36,009)\end{array}$ & Monthly $(\mathrm{n}=90,639)$ & $\begin{array}{l}1-3 \text { days/week } \\
(n=146,289)\end{array}$ & $\begin{array}{l}\geq 4 \text { days/week } \\
(n=27,915)\end{array}$ & Overall $(n=300,852)$ \\
\hline Age in years, mean (SD) & $51.7(10.6)$ & $51.1(10.5)$ & $50.6(10.4)$ & $51.2(10.5)$ & $50.9(10.5)$ \\
\hline Urban $(\%)$ & 26.5 & 32.6 & 55.1 & 50.4 & 44.5 \\
\hline Education $>6$ years $(\%)$ & 36.6 & 39.8 & 45.8 & 52.7 & 43.3 \\
\hline Household income $>20,000$ yuan/year $(\%)$ & 31.2 & 37.2 & 42.3 & 47.9 & 40.7 \\
\hline Never smoker $(\%)$ & 94.2 & 94.7 & 95.3 & 95.2 & 94.9 \\
\hline Weekly alcohol consumption (\%) & 2.0 & 2.0 & 2.1 & 2.5 & 2.1 \\
\hline Adult attained height in $\mathrm{cm}$, mean (SD) & $153.6(6.1)$ & $153.9(6.0)$ & $154.3(5.9)$ & $154.8(5.8)$ & $154.1(6.0)$ \\
\hline $\mathrm{BMI}$ in $\mathrm{kg} / \mathrm{m}^{2}$, mean $(\mathrm{SD})$ & $23.7(3.6)$ & $23.7(3.4)$ & $23.9(3.4)$ & $23.9(3.5)$ & $23.8(3.5)$ \\
\hline Physical activity in MET-hour/day, mean (SD) & $20.3(12.0)$ & $20.4(12.5)$ & $20.5(12.9)$ & $20.5(13.4)$ & $20.5(12.8)$ \\
\hline Age at menarche in years, mean (SD) & $15.6(2.1)$ & $15.5(2.0)$ & $15.4(1.9)$ & $15.3(1.9)$ & $15.4(2.0)$ \\
\hline Parity, mean (SD) & $2.3(1.5)$ & $2.3(1.5)$ & $2.2(1.3)$ & $2.1(1.2)$ & $2.2(1.4)$ \\
\hline Months of breast-feeding per child, mean (SD) & $14.5(9.0)$ & $14.6(8.1)$ & $14.4(7.0)$ & $14.1(6.8)$ & $14.5(7.8)$ \\
\hline Post-menopause at baseline (\%) & 52.6 & 52.5 & 52.2 & 52.3 & 52.4 \\
\hline Ever use of oral contraceptives (\%) & 9.1 & 9.6 & 10.1 & 9.5 & 9.8 \\
\hline Family history of cancer (\%) & 16.3 & 16.5 & 16.6 & 17.7 & 16.6 \\
\hline \multicolumn{6}{|l|}{ Regular consumption of foods ${ }^{b}$} \\
\hline Fresh fruit & 23.7 & 25.6 & 34.5 & 44.9 & 31.7 \\
\hline Fresh vegetables & 97.7 & 97.8 & 99.0 & 99.2 & 98.3 \\
\hline Preserved vegetables & 24.9 & 21.8 & 22.6 & 25.4 & 22.8 \\
\hline Red meat & 36.6 & 40.1 & 45.9 & 51.6 & 44.1 \\
\hline Poultry & 0.7 & 0.7 & 0.9 & 2.4 & 1.0 \\
\hline Fish & 6.2 & 7.9 & 8.5 & 12.9 & 8.5 \\
\hline Dairy & 8.5 & 9.2 & 13.3 & 19.7 & 12.5 \\
\hline Total energy intake in $\mathrm{kcal} / \mathrm{day}$, median & 1078.6 & 1139.3 & 1256.6 & 1280.1 & 1231.8 \\
\hline
\end{tabular}

${ }^{a}$ Values for all variable except age, urban and total energy intake were standardized for age and region

${ }^{b}$ Participants eating foods for at least 4 days per week were regarded as regular consumers 
a regular consumption (i.e. $\geq 4$ days/week). The estimated mean (SD) value of usual soy isoflavone intake was 9.4 (5.2) $\mathrm{mg} /$ day, corresponding to 7.5 (4.2) $\mathrm{g} /$ day of soybean equivalents. The spearman coefficient between baseline frequency group and usual amount quartile of soy intake was 0.76 . Women consuming soy more frequently were more likely to live in urban areas, to have a higher level of education, and higher household income. They were also more likely to be taller, to have a family history of cancer, to be regular consumers of fresh fruit, red meat, fish and dairy products, and to have higher total energy intake. There were no obvious variation in age, BMI, level of physical activity and reproductive characteristics between soy consumption categories. Few of the women were ever smoker or weekly alcohol consumers.

\section{Association of soy consumption with incident breast cancer}

During a mean follow-up of 10 years, 2289 women developed incident breast cancer. Among the 2,289 breast cancer cases, 1120 were identified among premenopausal women and 1169 among postmenopausal women at baseline. The mean (SD) age at breast cancer diagnosis was $56.6(9.8)$ years. Table 2 shows the risk of incident breast cancer in relation to the frequency and usual amount of soy isoflavone intake. Overall, there was no evidence of any association between soy intake and breast cancer risk. Comparing with never/rare consumers, the multivariable-adjusted hazard ratios were 1.03 (95\% CI 0.87-1.22) for women consuming soy foods 1-3 days per week and 0.98 (95\% CI 0.80-1.22) for regular consumers. The trend tests were not statistically significant. Likewise, risk estimates were similar across quartiles of soy isoflavone intake in the total population.

Baseline menopausal status did not appear to modify the results (Table 3, $P=0.856$ for frequency-menopausal status interaction and $P=0.972$ for quartile-menopausal status interaction). In the subgroup analysis according to BMI, higher soy intake seemed to be inversely associated the risk of breast cancer in women with BMI $<24 \mathrm{~kg} / \mathrm{m}^{2}$, with a HR $(95 \% \mathrm{CI})$ of $0.76(0.60-0.96)$ for the highest soy frequency consumption group and a HR $(95 \% \mathrm{CI})$ of 0.75 (0.54-1.04) for the highest quartile of soy consumption (Appendix Table 2). But no statistically significant interaction between soy intake and BMI was detected (Appendix Table 2: $P=0.131$ for frequency-BMI interaction and $P=0.281$ for quartile-BMI interaction; Appendix Tables 3 and 4). In sensitivity analyses, neither the exclusion of cases

Table 2 Association of soy consumption and risk of incident breast cancer: multivariable Cox models

\begin{tabular}{|c|c|c|c|c|c|c|}
\hline & \multirow[t]{2}{*}{ No. of cases } & \multirow[t]{2}{*}{ Person-years } & \multirow{2}{*}{$\begin{array}{l}\text { Incidence rate (per } \\
100,000 \text { person- } \\
\text { years) }^{b}\end{array}$} & \multicolumn{3}{|c|}{ Adjusted hazard ratio (95\% confidence intervals) } \\
\hline & & & & Model 1 & Model 2 & Model 3 \\
\hline \multicolumn{7}{|l|}{ Baseline soy frequency } \\
\hline Never or rarely (Median 3.2 mg/day) & 194 & 359,459 & 66.0 & 1.00 & 1.00 & 1.00 \\
\hline Monthly (Median 4.5 mg/day) & 546 & 904,889 & 75.2 & $1.11(0.94-1.31)$ & $1.10(0.93-1.31)$ & $1.08(0.91-1.28)$ \\
\hline 1-3 days/week (Median $14.4 \mathrm{mg} /$ day) & 1291 & $1,471,813$ & 77.7 & $1.09(0.92-1.28)$ & $1.07(0.91-1.27)$ & $1.03(0.87-1.22)$ \\
\hline$\geq 4$ days/week (Median $19.1 \mathrm{mg} /$ day) & 258 & 279,390 & 78.7 & $1.05(0.86-1.29)$ & $1.03(0.84-1.26)$ & $0.98(0.80-1.20)$ \\
\hline$P$ for trend ${ }^{\mathrm{a}}$ & & & & 0.817 & 0.998 & 0.537 \\
\hline \multicolumn{7}{|l|}{ Quartiles of usual soy isoflavone intake } \\
\hline Q1 (Median 4.5 mg/day) & 495 & 993,841 & 67.1 & 1.00 & 1.00 & 1.00 \\
\hline Q2 (Median $7.2 \mathrm{mg} /$ day) & 311 & 517,016 & 70.9 & $0.99(0.84-1.17)$ & $0.99(0.83-1.17)$ & $0.96(0.81-1.13)$ \\
\hline Q3 (Median 14.4 mg/day) & 1,309 & $1,344,269$ & 80.9 & $1.11(0.97-1.28)$ & $1.10(0.96-1.26)$ & $1.06(0.92-1.22)$ \\
\hline Q4 (Median 19.1 mg/day) & 174 & 160,425 & 80.9 & $1.05(0.86-1.28)$ & $1.03(0.84-1.26)$ & $1.00(0.81-1.22)$ \\
\hline$P$ for trend ${ }^{\mathrm{a}}$ & & & & 0.288 & 0.379 & 0.682 \\
\hline
\end{tabular}

Model 1: Stratified by baseline age groups and study regions and adjusted for education attainment (no formal school, primary school, middle school, or high school or above) and household income (<10000, 10000-19999, or $\geq 20000$ yuan/year)

Model 2:Adjusted for variables in model 1 plus smoking status (never or ever), alcohol consumption (weekly alcohol consumer or non-weekly consumer), physical activity (0-10.9, 11.0-17.9, 18.0-29.4, or $\geq 29.5$ MET-hour/day), baseline BMI $\left(<24.0,24.0-27.9\right.$, or $\geq 28.0 \mathrm{~kg} / \mathrm{m}^{2} \mathrm{accord}-$ ing to overweight/obesity definition of Chinese population), and standing height (continuous)

Model 3: Adjusted for variables in model 2 plus age at menarche $(<10,11,12,13,14,15,16,17,18$, or $\geq 19$ years old), parity ( 0 , 1, 2, or $\geq 3$ ), average breastfeeding duration $(0,0.1-1,1-2$, or $>2$ years), menopausal status and age at menopause (pre-menopausal, menopausal age $<45$ years old, menopausal age 45-49 years old, menopausal age $\geq 50$ years old), use of oral contraceptives (ever or never), family history of cancer (yes or no), consumption frequency of fresh fruit, fresh vegetables, preserved vegetables, red meat, poultry, fish and dairy products at baseline (never/rarely, monthly, 1-3 days/week, 4-6 days/week, or daily), and total energy intake

${ }^{\mathrm{a}}$ Tests for trend were conducted by coding the groups as 1,2, 3 and 4, respectively

${ }^{\mathrm{b}}$ Values were adjusted for study region and age at study date 
Table 3 Soy intake and risk of incident breast cancer according to menopausal status

\begin{tabular}{|c|c|c|c|c|}
\hline & No. of cases & Person-years & $\begin{array}{l}\text { Incidence rate (per } \\
100,000 \text { person-years) }\end{array}$ & $\begin{array}{l}\text { Adjusted hazard ratio } \\
\text { ( } 95 \% \text { confidence } \\
\text { intervals) }^{\text {b }}\end{array}$ \\
\hline \multicolumn{5}{|c|}{ Baseline frequency $(P$ for interaction $=0.856)$} \\
\hline \multicolumn{5}{|c|}{ Pre-menopause } \\
\hline Monthly or less ${ }^{\mathbf{c}}$ & 376 & 629,714 & 73.6 & 1.00 \\
\hline 1-3 days/week & 628 & 709,199 & 78.9 & $0.99(0.85-1.15)$ \\
\hline$\geq 4$ days/week & 116 & 127,684 & 76.8 & $0.90(0.71-1.13)$ \\
\hline$P$ for trend ${ }^{\mathrm{d}}$ & & & & 0.433 \\
\hline \multicolumn{5}{|l|}{ Post-menopause } \\
\hline Monthly or less ${ }^{\mathbf{c}}$ & 364 & 634,634 & 72.4 & 1.00 \\
\hline 1-3 days/week & 663 & 762,614 & 76.3 & $0.94(0.81-1.09)$ \\
\hline$\geq 4$ days/week & 142 & 151,706 & 79.3 & $0.93(0.75-1.15)$ \\
\hline$P$ for trend ${ }^{\mathrm{d}}$ & & & & 0.446 \\
\hline \multicolumn{5}{|c|}{ Quartiles of usual soy isoflavone intake $(P$ for interaction $=0.972)$} \\
\hline \multicolumn{5}{|c|}{ Pre-menopause } \\
\hline Q1 & 233 & 452,731 & 66.4 & 1.00 \\
\hline Q2 & 157 & 229,605 & 73.5 & $1.00(0.79-1.28)$ \\
\hline Q3 & 652 & 706,875 & 81.8 & $1.11(0.91-1.35)$ \\
\hline Q4 & 78 & 77,386 & 80.6 & $1.00(0.74-1.35)$ \\
\hline$P$ for trend ${ }^{\mathrm{e}}$ & & & & 0.556 \\
\hline \multicolumn{5}{|l|}{ Post-menopause } \\
\hline Q1 & 262 & 541,110 & 68.9 & 1.00 \\
\hline Q2 & 154 & 287,411 & 70.4 & $0.89(0.70-1.13)$ \\
\hline Q3 & 657 & 637,395 & 79.0 & $0.99(0.81-1.21)$ \\
\hline Q4 & 96 & 83,039 & 79.3 & $0.97(0.73-1.28)$ \\
\hline$P$ for trend $\mathrm{d}^{\mathrm{e}}$ & & & & 0.872 \\
\hline
\end{tabular}

${ }^{\mathrm{a}}$ Values were adjusted for study region and age at study date

${ }^{\mathrm{b}}$ Adjusted for the same variables as model 3 in Table 2, except menopausal status

'The "Never or rarely" and "Monthly" groups were combined into the "Monthly or less" to ensure enough cases in each frequency category

'Test for trend was conducted by coding the "Monthly or less" group as 1, "1-3 days/week" group as 2, and " $\geq 4$ days/week" group as 3

${ }^{\mathrm{e}}$ Test for trend was conducted by coding from the lowest to the highest quartile into 1, 2, 3 and 4, respectively

occurring during the first 2 years of follow-up, nor excluding those with a family history of cancer materially altered the results (data not shown).

\section{Systematic review and dose-response meta-analysis}

A total of 452 records were retrieved from Pubmed, Embase, Cochrane library and through hand searching. After removing duplicated records and abstract screening, 28 full-text articles were reviewed for eligibility checking (Appendix Figure 1). Of these, 11 studies were eligible with their characteristics and results of quality assessment presented in Appendix Tables 5 and 6.

In the dose-response meta-analysis, we further excluded three studies $[15,18,20]$ because of markedly lower soy isoflavone intake when compared with other studies. The other eight studies were included in the present dose-response meta-analysis together with the CKB study, with a total of 631,498 women and 10,229 breast cancer cases. Seven [25, 26, 28, 30-32, 36] out of the eight studies used validated FFQs to assess soy intake while the FFQ in only one study [27] was not validated. Five studies [25, 26, 30-32] assessed both frequency and amount of soy food intake at baseline and calculated soy isoflavone intake based on the isoflavone content of different soy foods obtained from Food Composition Tables or published data. Two studies assessed soy food frequency at baseline and the portion size and isoflavone content were estimated from the validation study $[28,36]$. One study collected information on soy food frequency and the amount of soy isoflavone intake was not available from either the baseline survey or the validation 
study [27]. Seven out of the eight individual studies included in the present dose-response meta-analysis adjusted for total

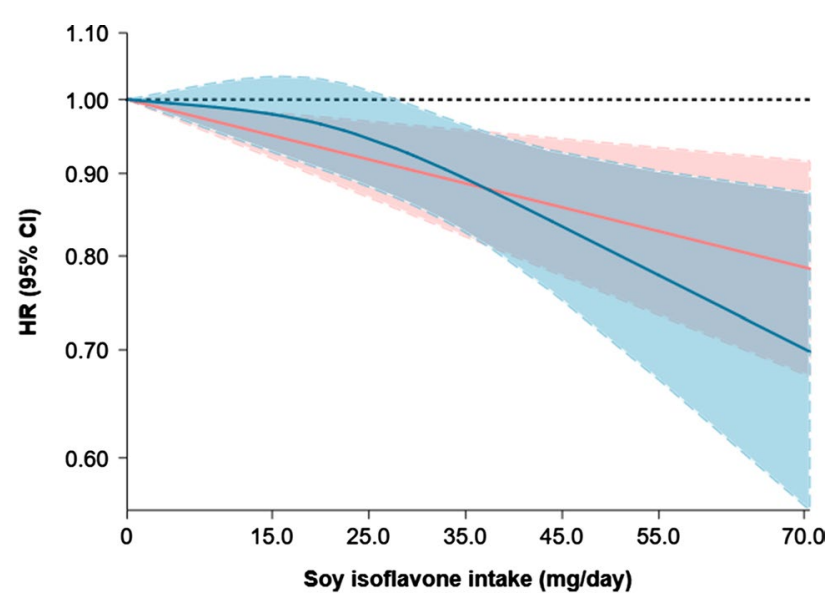

Fig. 1 The relationship between incident breast cancer and soy isoflavone intake in dose-response meta-analysis of CKB and 8 published cohorts. Test for non-linear dose-response relation between soy isoflavone intake and incident breast cancer was statistically insignificant ( $P$ for non-linearity $=0.142$ ). The blue line and shadow represent the hazard ratio (HR) and 95\% confidence interval (CI) for the non-linear model while the red line (shadow) represents the HRs (95\% CI) for the linear model energy intake when assessing the soy-breast cancer association [25, 26, 28, 30-32, 36]. Seven [25, 26, 28, 30-32, 36] out of the eight studies were of high and one study [27] of moderate quality according to the Newcastle-Ottawa quality assessment scale (NOS) scores (Appendix Table 6).

The dose-response meta-analysis using a RCS function failed to reject the linearity assumption between soy isoflavone intake and risk of breast cancer ( $P$ for non-linearity $=0.142$; Fig. 1). Linear dose-response meta-analysis found that each $10 \mathrm{mg} /$ day increment in soy isoflavone intake was associated with a mild reduced risk of breast cancer (HR: 0.97, 95\% CI 0.95-0.99; Fig. 2). The betweenstudy heterogeneity was low $\left(\mathrm{I}^{2}=19.8 \%, P\right.$ for heterogeneity $=0.267)$. The Egger test including the 9 studies in the dose-response meta-analysis found no publication bias $(P=0.606)$.

Sensitivity analyses by excluding one of the nine studies each time from the dose-response meta-analysis found that the risk estimates remained stable (Appendix Table 7). Sensitivity analysis by excluding two studies $[27,28]$ which reported HRs (95\% CIs) according to soy frequency rather than soy intake amount revealed no major change in risk estimate (HR: $0.96,95 \%$ CI $0.94-0.98 ; \mathrm{I}^{2}=22.1 \%, P$ for heterogeneity $=0.260$ ). Sensitivity analysis by including the five studies [25, 26, 30-32] that assessed both frequency and

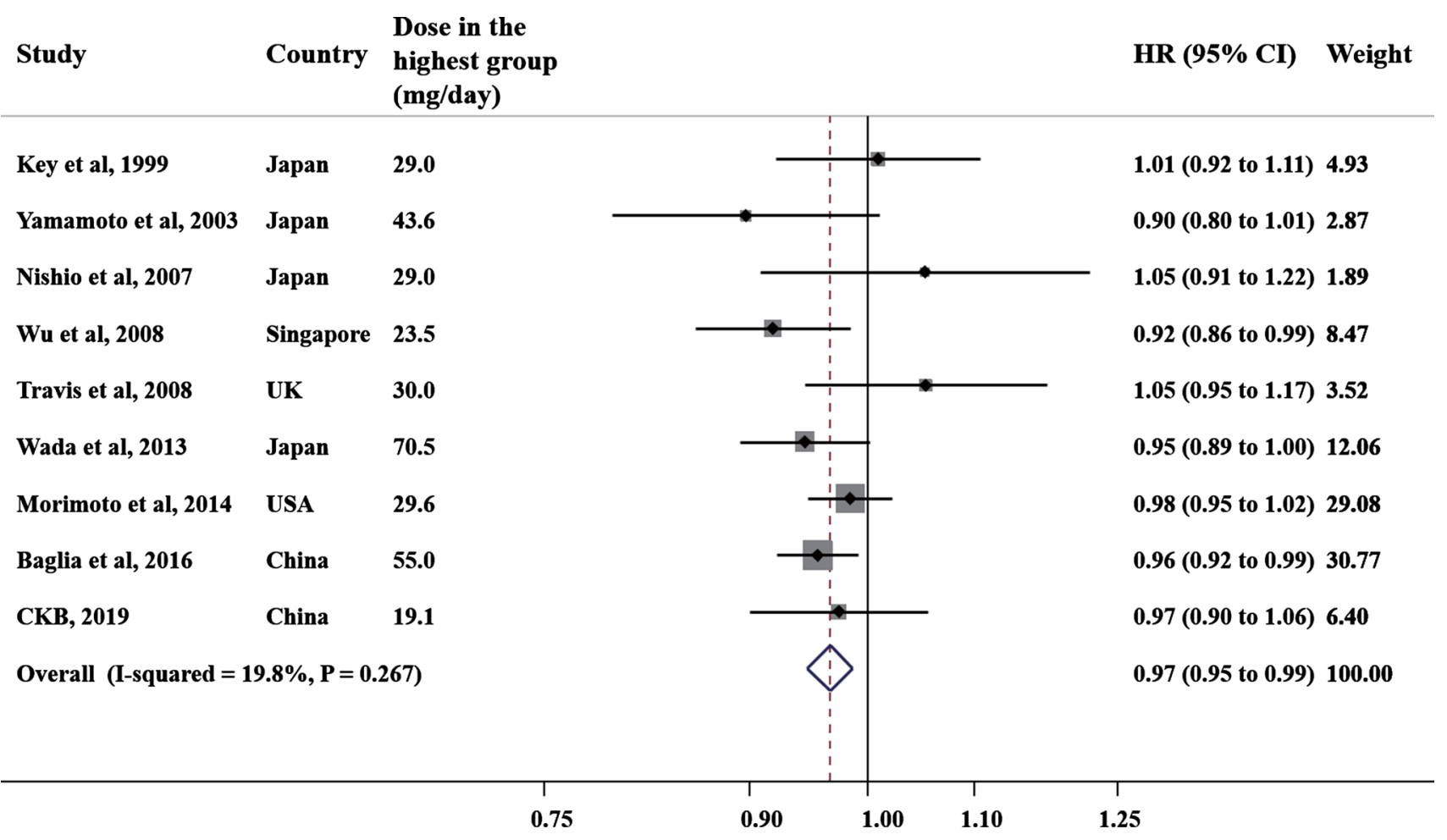

Fig. 2 The forest plot of hazard ratio (HR) and 95\% confidence interval (CI) of incident breast cancer for each $10 \mathrm{mg} /$ day increment in soy isoflavone intake in dose-response meta-analysis of CKB and 8 published cohorts (fixed effect model). The sizes of square boxes are inversely proportional to the variances of logarithmic HRs 
amount of soy intake at baseline found similar risk estimate as the main dose-response meta-analysis (HR: 0.97, 95\% CI $0.94-0.99 ; \mathrm{I}^{2}=35.9 \%, P$ for heterogeneity $=0.182$ ). The dose-response meta-analysis of all 12 studies gave the same risk estimate (HR: $0.97,95 \%$ CI $0.95-0.99 ; \mathrm{I}^{2}=0.0 \%, P$ for heterogeneity $=0.499$ ) as the main dose-response metaanalysis of nine studies.

\section{Discussion}

The CKB study, which enrolled over 300,000 women from 10 diverse regions from China, found no association between soy intake and incident breast cancer overall. The dose-response meta-analysis integrating the CKB study and other prospective studies from Asia and Western countries found that each $10 \mathrm{mg} /$ day of soy isoflavone intake was associated with a $3 \%$ reduced breast cancer risk.

Findings from prospective studies on soy-breast cancer association were inconsistent, which may be due to different soy intake levels across different studies. Cohort studies conducted among women with low $(<5 \mathrm{mg}$ /day in the highest consumption groups) $[15,18,20]$ or moderate $(20-30 \mathrm{mg} /$ day in the highest consumption groups) [25-28] soy isoflavone intake found no clear association between soy and breast cancer risk. In the CKB study, the median amount of soy isoflavone intake in the highest consumption group was about $20 \mathrm{mg} /$ day and no association was found between soy intake and breast cancer risk. This result was consistent with the four cohorts with moderate amount of soy intake [25-28]. In four studies in which the highest quartile or quintile intake groups had soy isoflavone $>40 \mathrm{mg} /$ day $[31,32,36]$ or the upper half intake group had a median intake at $23.5 \mathrm{mg} /$ day (so upper quartile group is likely to have $\sim 40 \mathrm{mg}$ /day) [30]), reduced breast cancer risk was found for women among the highest soy consumption group compared to women among the lowest consumption group.

The soy intake level (mean: $7.5 \mathrm{~g} / \mathrm{day}$ of soybean equivalents) was much lower among the CKB women than that assessed in the study conducted in Shanghai, China (median $\sim 25 \mathrm{~g} /$ day of soybean equivalents) [32], which may be partially explained by the relatively lower level of soy intake among general Chinese adults than that among women in Shanghai. According to the three Chinese National Nutrition and Health Surveys conducted between 1992 and 2012, the mean daily intake of soy foods among Chinese adults was about $10-15 \mathrm{~g}$ of soybean equivalents, and remained stable throughout the past two decades [37]. The mean daily soy intake was slightly lower among CKB women compared with results from the three Chinese national nutrition and health surveys, while the average soy intake in women of the Shanghai study was much higher than general Chinese population.
We found an inverse association between higher soy food frequency and breast cancer among CKB women with lower BMI while no statistically significant association was observed among women with higher BMI. The Singapore Chinese study also reported different risks estimates among women with different BMI [30]. However, tests for multiplicative interaction between soy intake and BMI were statistically insignificant in both studies. Therefore, it is likely that different risk estimates for soy intake among women with different body sizes were due to chance.

The present dose-response meta-analysis observed a $3 \%$ (95\% CI 1-5\%) reduced risk of breast cancer for each $10 \mathrm{mg} /$ day increment in soy isoflavone intake. Most of the cohort studies included in the present dose-response meta-analysis used validated FFQs to assessed soy intake. Most studies were of high quality according to the NOS scores which evaluated studies in terms of exposure and outcome measurement, confounding adjustment, followup duration and so on. Sensitivity analysis by excluding the study [27] which was of lower quality observed no change in the risk estimate, and sensitivity analysis by including exclusively the five studies that assessed both the frequency and amount of soy intake at baseline gave similar risk estimate, indicating the robustness of the present dose-response meta-analysis. Besides, between-study heterogeneity was low in the main dose-response metaanalysis as well as in sensitivity analyses, and the Egger test found no evidence of publication bias, indicating the reliability of the dose-response meta-analysis. In the present dose-response meta-analysis, the breast cancer risk reduced by each $10 \mathrm{mg} /$ day of soy isoflavone intake as weakly as $3 \%$, which may explain why most individual studies (including CKB) with low to moderate level of soy isoflavone intake failed to detect a statistically significant soy-breast cancer association.

According to the Dietary Guidelines for Chinese Residents in 2016, the recommended daily amount of soy food intake for adults were 15-25 g soybean equivalents [37]. The isoflavone content for 15-25 g/day soybean equivalents of different soy foods range between 10 and $50 \mathrm{mg}$ /day (Appendix Table 8). Therefore, women consuming soy foods at the amount recommended by the Dietary Guidelines may be at $3-15 \%$ reduced risk of breast cancer according to the result of the present dose-response meta-analysis.

Could soy isoflavone supplement help? Two Western studies assessed soy supplement or soy isoflavone supplement, and neither of them found association of soy or soy isoflavone supplement with overall breast cancer risk [38, 39], though one study found that current soy isoflavone supplement was associated with reduced risk of estrogen receptor positive $(\mathrm{ER}+)$ breast cancer and increased risk of ER- breast cancer [39]. However, the dose of isoflavone in the supplements could vary in the study [39]. 
As the largest cohort study on soy-breast cancer association, the CKB study has several strengths, including large sample size, population from diverse areas across China, prospective cohort design, long duration of follow-up, unified method of exposure assessment across study regions, and stringent quality control of data. Our meta-analysis included only prospective cohort studies, which minimized recall bias.

There are several limitations, nevertheless. Firstly, the baseline questionnaire asked CKB participants about soy intake frequency rather than intake amount, and thus the usual amount of soy isoflavone was estimated by combing information from baseline surveys, two resurveys and the 24-HDRs. More large scale prospective studies using more precise exposure measurement methods are warranted to verify findings from the CKB study. Secondly, some energyproviding food items such as oil were not assessed in the CKB study, and therefore the calculated total energy was lower than the actual total energy intake. However, the risk estimates in the CKB study remained stable before and after adjustment for the calculated total energy intake. Thirdly, data on hormone receptor status of breast cancer were unavailable in the CKB study, so we were unable to assess the association of soy intake with breast cancer subtypes. Existing prospective evidence of soy-breast cancer subtype association has been scarce and of low statistical power due to small sample sizes [30, 32, 39]. Lastly, owing to the observational nature of studies included in the dose-response metaanalysis, we could not rule out the possibility of residual confounding caused by unmeasured factors. Large randomized controlled trials (RCT) may be desirable in causal inference but the feasibility of a large RCT on this issue is still a sticky issue.

\section{Conclusion}

The CKB study demonstrated that moderate soy intake was not associated with breast cancer risk among Chinese women. Higher amount of soy intake might provide reasonable benefits for the prevention of incident breast caner. More large scale and well-designed prospective studies are needed to verify our findings.

\footnotetext{
Acknowledgements The chief acknowledgment is to the participants, the project staff, and the China National Centre for Disease Control and Prevention (CDC) and its regional offices for assisting with the fieldwork. We thank Judith Mackay in Hong Kong; Yu Wang, Gonghuan Yang, Zhengfu Qiang, Lin Feng, Maigeng Zhou, Wenhua Zhao, and Yan Zhang in China CDC; Lingzhi Kong, Xiucheng Yu, and Kun $\mathrm{Li}$ in the Chinese Ministry of Health; and Sarah Clark, Martin Radley, Mike Hill, Hongchao Pan, and Jill Boreham in the CTSU, Oxford, for assisting with the design, planning, organization, and conduct of the study. We sincerely appreciate Chenxi Qin for her help and support in
}

issues about dietary assessment. We thank Gertraud Maskarinec and $\mathrm{AH} \mathrm{Wu}$ for providing data for the present dose-response meta-analysis.

Contributors

Canqing Yu and Dezheng Huo conceived and designed the study. Liming Li, Zhengming Chen, and Junshi Chen, as the members of CKB steering committee, designed and supervised the conduct of the whole study, obtained funding, and together with Jun Lv, Yu Guo, Zheng Bian, Huaidong Duo, Ling Yang, Yiping Chen, Xi Zhang and Tao Wang authors acquired the data. Yuxia Wei and Meng Gao analyzed the data. Yuxia Wei wrote the first draft of the manuscript. CanqingYu and Dezheng Huo contributed to the interpretation of the results and critical revision of the manuscript for important intellectual content. All authors reviewed and approved the final manuscript. Canqing $\mathrm{Yu}$ and Dezheng Huo are the guarantors.

Members of the China Kadoorie Biobank collaborative group International Steering Committee: Junshi Chen, Zhengming Chen (PI), Robert Clarke, Rory Collins, Yu Guo, Liming Li (PI), Jun Lv, Richard Peto, Robin Walters. International Co-ordinating Centre, Oxford: Daniel Avery, Ruth Boxall, Derrick Bennett, Yumei Chang, Yiping Chen, Zhengming Chen, Robert Clarke, Huaidong Du, Simon Gilbert, Alex Hacker, Mike Hill, Michael Holmes, Andri Iona, Christiana Kartsonaki, Rene Kerosi, Ling Kong, Om Kurmi, Garry Lancaster, Sarah Lewington, Kuang Lin, John McDonnell, Iona Millwood, Qunhua Nie, Jayakrishnan Radhakrishnan, Paul Ryder, Sam Sansome, Dan Schmidt, Paul Sherliker, Rajani Sohoni, Becky Stevens, Iain Turnbull, Robin Walters, Jenny Wang, Lin Wang, Neil Wright, Ling Yang, Xiaoming Yang. National Co-ordinating Centre, Beijing: Zheng Bian, Yu Guo, Xiao Han, Can Hou, Jun Lv, Pei Pei, Chao Liu, Yunlong Tan, Canqing Yu. 10 Regional Co-ordinating Centres: Qingdao CDC: Zengchang Pang, Ruqin Gao, Shanpeng Li, Shaojie Wang, Yongmei Liu, Ranran Du, Yajing Zang, Liang Cheng, Xiaocao Tian, Hua Zhang, Yaoming Zhai, Feng Ning, Xiaohui Sun, Feifei Li. Licang CDC: Silu Lv, Junzheng Wang, Wei Hou. Heilongjiang Provincial CDC: Mingyuan Zeng, Ge Jiang, Xue Zhou. Nangang CDC: Liqiu Yang, Hui He, Bo Yu, Yanjie Li, Qinai Xu,Quan Kang, Ziyan Guo. Hainan Provincial CDC: Dan Wang, Ximin Hu, Jinyan Chen, Yan Fu, Zhenwang Fu, Xiaohuan Wang. Meilan CDC: Min Weng, Zhendong Guo, Shukuan Wu, Yilei Li, Huimei Li, Zhifang Fu. Jiangsu Provincial CDC: Ming Wu, Yonglin Zhou, Jinyi Zhou, Ran Tao, Jie Yang, Jian Su. Suzhou CDC: Fang liu, Jun Zhang, Yihe Hu, Yan Lu, Liangcai Ma, Aiyu Tang, Shuo Zhang, Jianrong Jin, Jingchao Liu. Guangxi Provincial CDC: Zhenzhu Tang, Naying Chen, Ying Huang. Liuzhou CDC: Mingqiang Li, Jinhuai Meng, Rong Pan, Qilian Jiang, Jian Lan, Yun Liu, Liuping Wei, Liyuan Zhou, Ningyu Chen Ping Wang, Fanwen Meng, Yulu Qin, Sisi Wang. Sichuan Provincial CDC: Xianping Wu, Ningmei Zhang, Xiaofang Chen, Weiwei Zhou. Pengzhou CDC: Guojin Luo, Jianguo Li, Xiaofang Chen, Xunfu Zhong, Jiaqiu Liu, Qiang Sun. Gansu Provincial CDC: Pengfei Ge, Xiaolan Ren, Caixia Dong. Maiji CDC: Hui Zhang, Enke Mao, Xiaoping Wang, Tao Wang, Xi zhang. Henan Provincial CDC: Ding Zhang, Gang Zhou, Shixian Feng, Liang Chang, Lei Fan. Huixian CDC: Yulian Gao, Tianyou He, Huarong Sun, Pan He, Chen Hu, Xukui Zhang, Huifang Wu, Pan He. Zhejiang Provincial CDC: Min Yu, Ruying Hu, Hao Wang. Tongxiang CDC: Yijian Qian, Chunmei Wang, Kaixu Xie, Lingli Chen, Yidan Zhang, Dongxia Pan, Qijun Gu. Hunan Provincial CDC: Yuelong Huang, Biyun Chen, Li Yin, Huilin Liu, Zhongxi Fu, Qiaohua Xu. Liuyang CDC: Xin Xu, Hao Zhang, Huajun Long, Xianzhi Li, Libo Zhang, Zhe Qiu.

Funding This work was supported by Grants $(81530088,81390540$, 81390544, 81390541) from National Natural Science Foundation of China; (2016YFC0900500, 2016YFC0900501, 2016YFC0900504, 2016YFC1303904) from the National Key Research and Development Program of China; a grant from the Kadoorie Charitable Foundation in Hong Kong; Grants from the UK Wellcome Trust (202922/Z/16/Z, 
088158/Z/09/Z, 104085/Z/14/Z); Grants from Chinese Ministry of Science and Technology (2011BAI09B01); and Breast Cancer Research Foundation. The funders and sponsors of the study had no role in the study design and conduct, data collection, data analysis, data interpretation, writing of the report, or the decision to submit the manuscript for publication. The corresponding author had full access to all the data in the study and had final responsibility for the decision to submit for publication.

\section{Compliance with ethical standards}

Conflict of interest We declare that we have no conflicts of interest.

Ethical approval The Ethical Review Committee of the Chinese Center for Disease Control and Prevention (Beijing, China) and the Oxford Tropical Research Ethics Committee, University of Oxford (UK) approved the study.

Informed consent Informed consent was obtained from all individual participants included in the study.

Data sharing Details of how to access China Kadoorie Biobank data and details of the data release schedule are available from www. ckbiobank.org/site/Data+Access. The lead author affirms that the manuscript is an honest, accurate, and transparent account of the study being reported; that no important aspects of the study have been omitted; and that any discrepancies from the study as planned have been explained.

Open Access This article is distributed under the terms of the Creative Commons Attribution 4.0 International License (http://creativeco mmons.org/licenses/by/4.0/), which permits unrestricted use, distribution, and reproduction in any medium, provided you give appropriate credit to the original author(s) and the source, provide a link to the Creative Commons license, and indicate if changes were made.

\section{References}

1. Bray F, Ferlay J, Soerjomataram I, Siegel RL, Torre LA, Jemal A. Global cancer statistics 2018: GLOBOCAN estimates of incidence and mortality worldwide for 36 cancers in 185 countries. CA Cancer J Clin. 2018;68(6):394-424. https://doi. org/10.3322/caac.21492.

2. Ferlay J, Shin HR, Bray F, Forman D, Mathers C, Parkin DM. Estimates of worldwide burden of cancer in 2008: GLOBOCAN 2008. Int J Cancer. 2010;127(12):2893-917. https://doi. org/10.1002/ijc.25516.

3. DeSantis CE, Bray F, Ferlay J, Lortet-Tieulent J, Anderson $\mathrm{BO}$, Jemal A. International variation in female breast cancer Incidence and mortality rates. Cancer Epidem Biomar. 2015;24(10):1495-506. https://doi.org/10.1158/1055-9965. EPI-15-0535.

4. Parkin DM, Pisani P, Ferlay J. Global cancer statistics. CA Cancer J Clin. 1999;49(1):33-64.

5. Parkin DM. Global cancer statistics in the year 2000. Lancet Oncol. 2001;2(9):533-43. https://doi.org/10.1016/s1470 -2045(01)00486-7.

6. Uifalean A, Schneider S, Ionescu C, Lalk M, Iuga CA. Soy isoflavones and breast cancer cell lines: molecular mechanisms and future perspectives. Molecules (Basel, Switzerland). 2015;21(1):E13. https://doi.org/10.3390/molecules21010013.
7. Huser S, Guth S, Joost HG, Soukup ST, Kohrle J, Kreienbrock $\mathrm{L}$, et al. Effects of isoflavones on breast tissue and the thyroid hormone system in humans: a comprehensive safety evaluation. Arch Toxicol. 2018;92(9):2703-48. https://doi.org/10.1007/ s00204-018-2279-8.

8. World Cancer Research Fund/American Institute for Cancer Research. Continuous Update Project Expert Report 2018. Diet, nutrition, physical activity and breast cancer. 2018.

9. Dong JY, Qin LQ. Soy isoflavones consumption and risk of breast cancer incidence or recurrence: a meta-analysis of prospective studies. Breast Cancer Res Treat. 2011;125(2):315-23. https:// doi.org/10.1007/s10549-010-1270-8.

10. Xie Q, Chen ML, Qin Y, Zhang QY, Xu HX, Zhou Y, et al. Isoflavone consumption and risk of breast cancer: a dose-response meta-analysis of observational studies. Asia Pac J Clin Nutr. 2013;22(1):118-27. https://doi.org/10.6133/apjen.2013.22.1.16.

11. Chen M, Rao Y, Zheng Y, Wei S, Li Y, Guo T, et al. Association between soy isoflavone intake and breast cancer risk for pre- and post-menopausal women: a meta-analysis of epidemiological studies. PLoS ONE. 2014;9(2):e89288. https://doi.org/10.1371/journ al.pone.0089288.

12. Bahrom S, Idris NRN, editors. Soy intake and breast cancer risk: a meta-analysis of epidemiological studies. In: Aip conference; 2016.

13. Zhao TT, Jin F, Li JG, Xu YY, Dong HT, Liu Q, et al. Dietary isoflavones or isoflavone-rich food intake and breast cancer risk: a meta-analysis of prospective cohort studies. Clin Nutr. 2019;38(1):136-45. https://doi.org/10.1016/j.clnu.2017.12.006.

14. den Tonkelaar I, Keinan-Boker L, Veer PV, Arts CJ, Adlercreutz $\mathrm{H}$, Thijssen $\mathrm{JH}$, et al. Urinary phytoestrogens and postmenopausal breast cancer risk. Cancer Epidemiol Biomark Prevent Publ Am Assoc Cancer Res cospons Am Soc Prevent Oncol. 2001;10(3):223-8.

15. Horn-Ross PL, Hoggatt KJ, West DW, Krone MR, Stewart SL, Anton $\mathrm{H}$, et al. Recent diet and breast cancer risk: the California Teachers Study (USA). Cancer Causes Control. 2002;13(5):407-15.

16. Grace PB, Taylor JI, Low YL, Luben RN, Mulligan AA, Botting NP, et al. Phytoestrogen concentrations in serum and spot urine as biomarkers for dietary phytoestrogen intake and their relation to breast cancer risk in European prospective investigation of cancer and nutrition-norfolk. Cancer Epidemiol Biomark Prevent Publ Am Assoc Cancer Res cospons Am Soc Prevent Oncol. 2004;13(5):698-708.

17. Keinan-Boker L, van Der Schouw YT, Grobbee DE, Peeters PH. Dietary phytoestrogens and breast cancer risk. Am J Clin Nutr. 2004;79(2):282-8. https://doi.org/10.1093/ajcn/79.2.282.

18. Touillaud MS, Thiebaut AC, Niravong M, Boutron-Ruault MC, Clavel-Chapelon F. No association between dietary phytoestrogens and risk of premenopausal breast cancer in a French cohort study. Cancer Epidemiol Biomark Prevent Publ Am Assoc Cancer Res cospons Am Soc Prevent Oncol. 2006;15(12):2574-6. https ://doi.org/10.1158/1055-9965.EPI-06-0543.

19. Verheus M, van Gils CH, Keinan-Boker L, Grace PB, Bingham SA, Peeters PH. Plasma phytoestrogens and subsequent breast cancer risk. J Clin Oncol. 2007;25(6):648-55. https://doi. org/10.1200/JCO.2006.06.0244.

20. Hedelin M, Lof M, Olsson M, Adlercreutz H, Sandin S, Weiderpass E. Dietary phytoestrogens are not associated with risk of overall breast cancer but diets rich in coumestrol are inversely associated with risk of estrogen receptor and progesterone receptor negative breast tumors in Swedish women. J Nutr. 2008;138(5):938-45.

21. Ward H, Chapelais G, Kuhnle GG, Luben R, Khaw KT, Bingham $\mathrm{S}$, et al. Breast cancer risk in relation to urinary and serum biomarkers of phytoestrogen exposure in the European 
Prospective into Cancer-Norfolk cohort study. Breast Cancer Res. 2008;10(2):R32. https://doi.org/10.1186/bcr1995.

22. Goodman MT, Shvetsov YB, Wilkens LR, Franke AA, Le Marchand L, Kakazu KK, et al. Urinary phytoestrogen excretion and postmenopausal breast cancer risk: the multiethnic cohort study. Cancer Prev Res (Phila). 2009;2(10):887-94. https://doi. org/10.1158/1940-6207.CAPR-09-0039.

23. Ward HA, Kuhnle GG, Mulligan AA, Lentjes MA, Luben RN, Khaw KT. Breast, colorectal, and prostate cancer risk in the European Prospective Investigation into Cancer and Nutrition-Norfolk in relation to phytoestrogen intake derived from an improved database. Am J Clin Nutr. 2010;91(2):440-8. https://doi.org/10.3945/ ajen.2009.28282.

24. Zamora-Ros R, Ferrari P, Gonzalez CA, Tjonneland A, Olsen A, Bredsdorff L, et al. Dietary flavonoid and lignan intake and breast cancer risk according to menopause and hormone receptor status in the European Prospective Investigation into Cancer and Nutrition (EPIC) Study. Breast Cancer Res Treat. 2013;139(1):163-76. https://doi.org/10.1007/s10549-013-2483-4.

25. Travis RC, Allen NE, Appleby PN, Spencer EA, Roddam AW, Key TJ. A prospective study of vegetarianism and isoflavone intake in relation to breast cancer risk in British women. Int J Cancer. 2008;122(3):705-10. https://doi.org/10.1002/ijc.23141.

26. Morimoto Y, Maskarinec G, Park SY, Ettienne R, Matsuno RK, Long C, et al. Dietary isoflavone intake is not statistically significantly associated with breast cancer risk in the Multiethnic Cohort. Br J Nutr. 2014;112(6):976-83. https://doi.org/10.1017/ S0007114514001780.

27. Key TJ, Sharp GB, Appleby PN, Beral V, Goodman MT, Soda $\mathrm{M}$, et al. Soya foods and breast cancer risk: a prospective study in Hiroshima and Nagasaki, Japan. Br J Cancer. 1999;81(7):124856. https://doi.org/10.1038/sj.bjc.6690837.

28. Nishio K, Niwa Y, Toyoshima H, Tamakoshi K, Kondo T, Yatsuya $\mathrm{H}$, et al. Consumption of soy foods and the risk of breast cancer: findings from the Japan Collaborative Cohort (JACC) Study. Cancer Causes Control. 2007;18(8):801-8. https://doi.org/10.1007/ s10552-007-9023-7.

29. Yamamoto S, Sobue T, Kobayashi M, Sasaki S, Tsugane S, Tsugane S, et al. Soy, isoflavones, and breast cancer risk in Japan. J Natl Cancer I. 2003;95(12):906-13. https://doi.org/10.1093/ jnci/95.12.906.

30. Wu AH, Koh WP, Wang R, Lee HP, Yu MC. Soy intake and breast cancer risk in Singapore Chinese Health Study. Br J Cancer. 2008;99(1):196-200. https://doi.org/10.1038/sj.bjc.6604448.
31. Wada K, Nakamura K, Tamai Y, Tsuji M, Kawachi T, Hori A, et al. Soy isoflavone intake and breast cancer risk in Japan: from the Takayama study. Int J Cancer. 2013;133(4):952-60. https:// doi.org/10.1002/ijc.28088.

32. Baglia ML, Zheng W, Li H, Yang G, Gao J, Gao YT, et al. The association of soy food consumption with the risk of subtype of breast cancers defined by hormone receptor and HER2 status. Int J Cancer. 2016;139(4):742-8. https://doi.org/10.1002/ijc.30117.

33. Chen Z, Chen J, Collins R, Guo Y, Peto R, Wu F, et al. China Kadoorie Biobank of 0.5 million people: survey methods, baseline characteristics and long-term follow-up. Int J Epidemiol. 2011;40(6):1652-66. https://doi.org/10.1093/ije/dyr120.

34. Yang G, Hu J, Rao KQ, Ma J, Rao C, Lopez AD. Mortality registration and surveillance in China: history, current situation and challenges. Popul Health Metr. 2005;3(1):3. https://doi. org/10.1186/1478-7954-3-3.

35. Greenland S, Longnecker MP. Methods for trend estimation from summarized dose-response data, with applications to meta-analysis. Am J Epidemiol. 1992;135(11):1301-9.

36. Yamamoto S, Sobue T, Kobayashi M, Sasaki S, Tsugane S. Soy, isoflavones, and breast cancer risk in Japan. J Natl Cancer I. 2003;95(12):906-13. https://doi.org/10.1093/jnci/95.12.906.

37. The Chinese Nutrition Society. The Dietary Guidelines for Chinese Residents (2016). Beijing, China: People's Medical Publishing House; 2016

38. Brasky TM, Lampe JW, Potter JD, Patterson RE, White E. Specialty supplements and breast cancer risk in the VITamins And Lifestyle (VITAL) Cohort. Cancer Epidemiol Biomark Prevent Publ Am Assoc Cancer Res cospons Am Soc Prevent Oncol. 2010;19(7):1696-708. https://doi.org/10.1158/1055-9965. EPI-10-0318.

39. Touillaud M, Gelot A, Mesrine S, Bennetau-Pelissero C, ClavelChapelon F, Arveux P, et al. Use of dietary supplements containing soy isoflavones and breast cancer risk among women aged $>50$ y: a prospective study. Am J Clin Nutr. 2019;109(3):597-605. https://doi.org/10.1093/ajen/nqy313.

Publisher's Note Springer Nature remains neutral with regard to jurisdictional claims in published maps and institutional affiliations.

\section{Affiliations}

\section{Yuxia Wei ${ }^{1}$. Jun $\mathrm{Lv}^{1,8,9} \cdot$ Yu Guo $^{2} \cdot$ Zheng Bian ${ }^{2} \cdot$ Meng Gao ${ }^{1} \cdot$ Huaidong Du ${ }^{3,4} \cdot$ Ling Yang $^{3,4} \cdot$ Yiping Chen $^{3,4}$. Xi Zhang ${ }^{5}$. Tao Wang ${ }^{5}$. Junshi Chen ${ }^{6}$. Zhengming Chen ${ }^{4}$. Canqing $\mathrm{Yu}^{1}$. Dezheng Huo ${ }^{7}\left(\mathrm{D} \cdot \mathrm{Liming} \mathrm{Li}^{1}\right.$ on behalf of the China Kadoorie Biobank Collaborative Group}

\author{
Yuxia Wei \\ 15260556916@163.com \\ Jun Lv \\ lvjun@bjmu.edu.cn \\ Yu Guo \\ guoyu@kscdc.net \\ Zheng Bian \\ bianzheng@kscdc.net \\ Meng Gao \\ gaom1995@163.com
}

\author{
Huaidong Du \\ huaidong.du@ndph.ox.ac.uk \\ Ling Yang \\ ling.yang@ndph.ox.ac.uk \\ Yiping Chen \\ yiping.chen@ndph.ox.ac.uk
}

Xi Zhang

936103510@qq.com

Tao Wang

895396379@qq.com 
Junshi Chen

chenjunshi@cfsa.net.cn

Zhengming Chen

zhengming.chen@ndph.ox.ac.uk

Liming Li

lmlee@vip.163.com

1 Department of Epidemiology and Biostatistics, School of Public Health, Peking University Health Science Center, 38 Xueyuan Road, Beijing 100191, China

2 Chinese Academy of Medical Sciences, Beijing, China

3 Medical Research Council Population Health Research Unit, University of Oxford, Oxford, UK

4 Clinical Trial Service Unit and Epidemiological Studies Unit (CTSU), Nuffield Department of Population Health, University of Oxford, Oxford, UK
5 NCDs Prevention and Control Department, Maiji CDC, Tianshui, Gansu, China

6 China National Center for Food Safety Risk Assessment, Beijing, China

7 Department of Public Health Sciences, The University of Chicago, 5841 S. Maryland Ave., MC2000, Chicago, IL 60637, USA

8 Key Laboratory of Molecular Cardiovascular Sciences (Peking University), Ministry of Education, Beijing, China

9 Peking University Institute of Environmental Medicine, Beijing, China 